

\section{DISCLAIMER}

This document was prepared as an account of work sponsored by the United States Goversment. Neither the United States Government nor any agency thereof, nor The Regents of the University of California, nor any of their employees, makes any warranty, express or implied, or assumes any legal liability or responsibility for the accuracy. completeness, or usefulness of iny information, apparatus, product, or process disclused, or represents that its use would not infringe privately owned rights. Reference herein to any specific commercial product, proccss, or service by its trade name, trademark, manufactures, or otherwise, does not necessarily constitute or imply its endorsement, recommendation, or favoring by the United States Coverrment or any agency thereol, or The Regents of the Uriversity of Califomia. The vicws and opinions of authors expressed herein do not necessarily state or reflect those of the United States Government or any agency thereof or The Regents of the University of Califomia and shall not be used for advertising or product endorsement purposes.

Lawrence Berkeley Laboratory is an equal spportunity employer. 


\title{
Flat Connection, Conformal Field Theory and Quantum Group*
}

\author{
Mitsuhiro Kato $\dagger$
}

\author{
Theoretical Physics Group \\ Physics Division \\ Laurence Berkeley Laboratory \\ 1 Cyclotron Road \\ Berkeley, CA 9\$720, USA
}

\begin{abstract}
General framework of linear first order diferential equation for four-point conformal block is studied by using flat connection. Integrability and $S L_{2}$ invariance restrict passible form of tlat connection. under a special ansatz classical Yang-Baxter equation appears as an integrability condition and the WZW model turns to be unique conformal field theory in that case. Monodromy property of conformal block can be easily determined by the flat connection.

\section{MASIER}

* Talk given at the XVIII-th International Conference on Differential Geometric Methods in The oretical Physics "Physics and Geometry" (July 3-8, Lake Tahoe). This work was supported by the Director, Office of Energy Research, Office of High Energy and Nuclear Physics, Division of High Energy Physics of the U.S. Department of Energy under Contract DE-AC03-76SF00098.

$\dagger$ On leave of absence from National Laboratory for High Energy Physics, Tsukuba, lbaraki 305, Japan
\end{abstract}




\title{
FLAT CONNECTION, CONFORMAL FIELD THEORY
}

\section{AND QUANTUM GROUP}

\author{
Mitsuhiro Kato $\dagger$ \\ Lawrence Berkeley Laboratory \\ University of California \\ Berkeley, CA 94720
}

\section{INTRODUCTION}

Recently many people ${ }^{1}$ are discussing general framework of rational conformal field theories (RCFT). There, one of the important concept is a connection matrix of conformal blocks. Once connection matrices are given for the conformal blocks, which are made from chiral vertex operators of a given chiral algebra by sandwiching them with $S L_{2}$ invariant vacuurn, then we can construct the physical correlation functions invariant under monodromy transformations and determine operator product expansion coefficients in principle.

The connection matrices satisfy some polynomial equations ${ }^{2}$ such as pentagon identity for example. Some people are trying to classify RCFT by solving the polynomial equations for connection matrices. The connection matrices, however, have only global information of conformal blocks. Moreover it is unclear how one can study dynamical or physical properties of the conformal blocks if playing only with the connection matrices.

On the other hand, we know that the conformal block satisfies a certain differential equation which is the result of null state structure of the representation. . $^{3,4}$ The differential equation contains both global and local information of the conformal block.

In the present note we will study the differential equations for conformal blocks in general framework. Especially we would like to point out the importance of flat connection. It may give a new and more tractable tool for classification of RCFT'.

$\dagger$ On leave of absence from National Laboratory for High Energy Physics, Tsukuba, Ibaraki 305, JAPAN 
Indeed for a special case we can completely classify the flat connections and hence CFT. Another advantage of using flat connection in writing down a differential equation is that a monodromy property of conformal block can be easily determined. In some case we can see quantum group structure for connection matrices without giving their explicit forms.

\section{DIFFERENTIAL EQUATIONS FOR CONFORMAL BLOCKS}

It is known that four-point function is the first nontrivial multi-point function in CFT. Let us assume a four-point function $\psi$ satisfy the following first order linear differential equation:

$$
\left(\frac{\partial}{\partial z_{i}}-w_{i}\right) \psi\left(z_{1}, z_{2}, z_{3}, z_{4}\right)=0 \quad i=1,2,3,4
$$

where $\psi$ is a $n$-column vector of functions and $w_{i}$ 's are $n \times n$ matrices of functions. In general, the number of fundamental solutions of (1), which are conformal blocks, is not greater than $n$.

Here one might wonder if differential equation for conformal block is always written in a first order form like (1). In the minimal model case, for instance, a correlation function (let us denote it by $f$ ) which contains primary field $\phi_{h_{(r .,)}}(z)$ is known to satisfy a differential equation of order $r s$ with respect to 2 . Even in this case we can define a rs-crilumn vector $\psi$ consists of $f, \frac{d}{d x} f, \cdots$, and $\left(\frac{d}{d x}\right)^{r s-1} f$ so that $\psi$ satisfies first ordes equation of the form (1). $W_{1}$ th similar tricks we can see that for every known differential equation of four-point function we can rewrite it in first order form (1). Hence (1) is most general form.

Now let us consider requirements for $w_{i}$. Among them two important things are (A) $S L_{2}$ invariance and (B) integrability.

(A) $S L_{2}$ invariance: Since we are considering CFT on a sphere, $S L_{2}$ invariance is one of necessary conditions. If we denote conformal dimension of each vertex operator by $\Delta_{i}(i=1,2,3,4)$, then $S L_{2}$ invariance of $\psi$ requires the following relations:

$$
\begin{gathered}
\sum_{i=1}^{4} \frac{\partial}{\partial z_{i}} \psi=0 \\
\sum_{i=1}^{4}\left(z_{i} \frac{\partial}{\partial z_{i}}+\Delta_{i}\right) \psi=0 \\
\sum_{i=1}^{4}\left(z_{i}^{2} \frac{\partial}{\partial z_{i}}+2 z_{i} \Delta_{i}\right) \psi=0
\end{gathered}
$$

Using eq. (1), we can derive the conditions for $\omega_{i}$ :

$$
\sum_{i=1}^{4} \omega_{i} \psi=0
$$




$$
\begin{gathered}
\sum_{i=1}^{4}\left(\Delta_{i}+z_{i} \omega_{i}\right) \psi=0 \\
\sum_{i=1}^{4}\left(2 z_{i} \Delta_{i}+z_{i}^{2} \omega_{i}\right) \psi=0
\end{gathered}
$$

(B) Integrability. Eq. (1) consists of four equations. So they have to be consistent with each other. This is achieved if differential operators $\frac{\partial}{\partial x_{i}}-\omega_{i}(i=1,2,3,4)$ cormmute with each other. Thus we have another set of conditions:

$$
\frac{\partial \omega_{j}}{\partial z_{i}}-\frac{\partial \omega_{i}}{\partial z_{j}}+\left[\omega_{i}, \omega_{j}\right]=0
$$

If you regard $\omega_{i}$ as a connection, (4) tells $\omega_{i}$ has zero curvature. It is very natural that a flat connection governs conformal block since analytic continuation of conformal block function does not depend on small (infinitesimal) deformation of continuation path. We will see later how the flat connection actually determine the connection matrix.

Besides the above conditions (A) and (B), we need several physical requirements to obtain differential equations corresponding to some RCFT. Unitarity, boundary condition at infinity and gauge choice are some of examples. Classification of RCFT based on this direction is now under investigation. Here we describe a remarkable trial of classification using a certain ansatz.

Let us assume $w_{i}$ has following form:

$$
\omega_{i}=\sum_{j \neq i} r_{i j}\left(z_{i j}\right)
$$

where $z_{i j}=z_{i}-z_{j}$ and $r_{i j}\left(z_{i j}\right)$ is a matrix of functions only depending on $z_{i j}$ satisfying $r_{i j}\left(z_{i j}\right)=-r_{j i}\left(z_{j i}\right)$. Then the integrability condition (4) becomes

$$
\left[r_{i j}\left(z_{i j}\right), r_{i k}\left(z_{i k}\right)\right]+\left[r_{i j}\left(z_{i j}\right), r_{j k}\left(z_{j k}\right)\right]+\left[r_{i k}\left(z_{i k}\right), r_{j k}\left(z_{j k}\right)\right]=0
$$

This equation is nothing but classical Yang-Baxter equation ${ }^{5}$ (CYBE)! Fortunately there exists a classification of its solutions by Belavin and Drinfel' $d^{6}$; a) rational, b) trigonometric and c) elliptic solutions. It can be easily seen that among them only rational solution satisfies $S L_{2}$ invariance. The rational solution has a form

$$
r_{i j}\left(z_{i j}\right)=\frac{\lambda}{2} \frac{1}{z_{i j}} \sum_{a} \rho_{i}\left(T^{a}\right) \otimes \rho_{j}\left(T^{a}\right)
$$

where $T^{\circ}$ 's are the orthonormal basis of arbitrary simple Lie algebra and $\rho_{i}$ is a certain representation. Normalization constant $\lambda$ can be fixed by another requirement such as unitarity. Substituting this expression into eq. (1), we obtain KrizhrikZarnolodchikov ${ }^{4}(\mathrm{KZ})$ equation.* This means the WZW model is a unique CFT

* Conversely, the fact that the integrability condition of $\mathrm{KZ}$ equation coincides with CYBE is first noted by Kohno?. 
under the ansatz (5). Although this ansatz does not include other interesting classes of CFT such as minimal model, the above discussion sufficiently exhibit powerfulness of this framework toward the classification of CFT.

\section{MONODROMY PROPERTIES OF CONFORMAL BLOCKS}

As mentioned before, an advantage of writing the diferential equation in the form of eq. (1) is that it enables us to write down a formal result of analytic continuation of solution. Let $\gamma$ be a path from a point $\left\{z_{i}\right\}$ to another point $\left\{z_{i}^{\prime}\right\}$ in $C^{4} \backslash \Delta$ (we denote $\Delta$ as a subspace of $C^{4}$ in which any two coordinates $z_{i}$ and $z_{j}$ coincide). Then the analytic continuation $\psi_{\gamma}\left(z_{i}^{\prime}\right)$ of $\psi\left(z_{i}\right)$ along $\gamma$ is expressed by a path-ordered integral:

$$
\psi_{\gamma}\left(z_{i}^{i}\right)=P_{\gamma} \exp \left(\int_{\left(x_{i}\right)}^{\left\{x_{i}^{\prime}\right)} \sum_{i} \omega_{j} d z_{j}\right) \psi\left(z_{i}\right)
$$

From this expression we can extract the property oi connection matrix. Before doing so, let us consider gaige degrees of freedom of the differential equation.

Eq. (1) has a gauge invariance (ve should rather say covariance) under the transformation

$$
\begin{aligned}
& \psi \rightarrow \psi^{\prime}=\mathcal{U} \psi \\
& \omega \rightarrow \omega^{\prime}=\mathcal{U} \omega \mathcal{U}^{-1}+(d \mathcal{U}) \mathcal{U}^{-1}
\end{aligned}
$$

where $\mathcal{U}\left(z_{1}, z_{2}, z_{3}, z_{4}\right)$ is a gauge transformation matrix and $\omega=\sum_{i} \omega_{i} d z_{i}$. What is more important is a subclass of gauge transformation which leaves $\omega$ invariant. The condition that $\mathcal{U}$ leaves $\omega$ invariant is

$$
d \mathcal{U}+[\mathcal{U}, \omega]=0
$$

Then $\mathcal{U} \psi$ satisfies the same differential equation as $\psi$ does, i.e. eq. (1), and can be expressed by a linear combination of fundamental solutions of (1). Defining a matrix $\Psi=\left(\psi^{(1)}, \psi^{(2)}, \cdots, \psi^{(n)}\right)$ which consists of fundamental solutions $\psi^{(i)}$, we can summarize the action of $\mathcal{U}$ in the following form:

$$
\mathcal{U} \Psi=\Psi U
$$

where $U$ is a constant matrix. $\ddagger$ We call $U$ that satisfies (10) monodromy operator $\S$ ard $U$ connection (monodromy) matrix.

Now let us consider the braiding operation for which we take a path $\gamma$ such that $z_{i}^{\prime}=z_{p(i)}$ where $p(i)$ is a permutation. The analytic continuation operator along $\gamma$

$$
T_{Y}=P_{\gamma} \exp \left(\int_{\left\{x_{i}\right\}}^{\left(x_{p(i)\}}\right)} \omega\right)
$$

$\ddagger$ We can easily see $d\left(\Psi^{-1} \mathcal{U} \Psi\right)=0$ by using the relations (10), $d \Psi=\omega \Psi$, and $d \Psi^{-1}=-\Psi^{-1} \omega$.

$\S$ Here the term monodramy is used somewhat extensively. 
becomes a matrix of functions of $z_{i}$. Generally $T_{\gamma}$ does not satisfy (10) unless $p(i)=i$. Instead,

$$
d T_{\gamma}=\omega\left(z_{p(i)}\right) T_{\gamma}-T_{\gamma} \omega\left(z_{i}\right)
$$

is held. If we find a constant matrix $\mathcal{A}$ which satisfies $\mathcal{A} \omega\left(z_{p(i)}\right) \mathcal{A}^{-1}=\omega\left(z_{i}\right)$, then $\mathcal{A} T_{\gamma}$ becomes a monodromy operator.

We take the WZW model for the illustration of above construction. In this case $\omega_{i}$ is given by eqs. (5) and (7), and $\psi$ is a function of four vectors $v_{i}$ of Lie algebra on which the representation $\rho_{i}\left(T^{a}\right)$ acts and four complex coordinates $z_{i}$. Taking an appropriate basis $\psi$ becomes a column vector. Now a braiding operator, say $\mathcal{B}_{23}$, is constructed as follows: First we obtain an analytic continuation operator $T_{\gamma}$ with an appropriate braiding path $\gamma$ for $z_{2}$ and $z_{3}$

$$
\psi_{\gamma}\left(v_{1} \otimes v_{2} \otimes v_{3} \otimes v_{4} ; z_{1}, z_{3}, z_{2}, z_{4}\right)=T_{\gamma} \psi\left(v_{1} \otimes v_{2} \otimes v_{3} \otimes v_{4} ; z_{1}, z_{2}, z_{3}, z_{4}\right)
$$

where

$$
T_{\gamma}=P_{\gamma} \exp \left(\int_{\left\{z_{1}, x_{2}, x_{3}, x_{4}\right\}}^{\left\{x_{1}, x_{3}, x_{2}, x_{4}\right\}} \omega\right)
$$

Second we find a constant matrix which turns $\omega\left(z_{1}, z_{3}, z_{2}, z_{4}\right)$ back to $\omega\left(z_{1}, z_{2}, z_{3}, z_{4}\right)$; the answer is permutation operator $\mathcal{P}_{23}$ of $v_{2} \otimes v_{3}$. Furthermore in this case we can explicitly perform the path-ordered integral and so the final result becomes

$$
\mathcal{B}_{23}=\mathcal{P}_{23} e^{i \pi \lambda \Omega_{33}}
$$

where $\Omega_{i j}=\frac{1}{2} \sum_{a} \rho_{i}\left(T^{a}\right) \otimes \rho_{j}\left(T^{a}\right)$.

We define a connection matrix $B_{i+1}$ corresponding to braid operation $\mathcal{B}_{i+1}$ according to the general story:

$$
\mathcal{B}_{i+1} \Psi=\Psi B_{i+1}
$$

Although the determination of $B$ itself is generally a hard task, much of its important properties can be obtained from the properties of $\mathcal{B}$ via eq. (17). Indeed $B$ and $\mathcal{B}$ have common properties such as eigenvalues and braid relation. The eigenvalues of $\mathcal{B}_{i j}$ are easily determined as $\epsilon_{i j}^{k} q^{c_{4}-c_{i}-c_{j}}$, where $c_{i}$ is Casimir invariant evaluated on the representation $\rho_{i}$ and $q=e^{i \pi \lambda}$. Index $k$ runs over the representations which appear in the decomposition $\rho_{i} \otimes \rho_{j}=\oplus_{k} \rho_{k}$, and $\epsilon_{i j}^{k}$ is either +1 or -1 depending on whether $\rho_{k}$ is constructed symmetrically or anti-symmetrically from $\rho_{i}$ and $\rho_{j}$.

In this line of argument, Kohno ${ }^{7}$ showed that the braiding matrices satisfy Hecke algebra if we choose $S L_{n}$ for Lie algebra and $n$-dimensional fundamental representation for every $\rho_{i}$. Furthermore we can show ${ }^{8,9}$ for the case of $S U_{2}$ that any connection matrices can be expressed by the $6 j$-symbols ${ }^{10}$ of quantum $\mathrm{SU}_{2}$ and the normalization factors for the conformal blocks. They completely agree with the explicit evaluation of the connection matrices by Tsuchiya and Kanie. ${ }^{11}$ 


\section{ACKNOWLEDGEMENTS}

We would like to thank P. Valtancoli for the collaboration from which this work was inspired. We have also benefited from discussions with T. Ozawa. This work was supported in part by the Director, Office of Energy Research, Office of High Energy and Nuclear Physics, Division of High Energy Physics of the U. S. Department of Energy under Contract DE-AC 03-76SF00098.

\section{REFERENCES}

1. See, for example, references listed in G. Moore and N. Seiberg, Classical and quantum conformal field theory, Commun. Math. Phys. 123:177 (1989).

2. G. Moore and N. Seiberg, Polynomial equations for rational conformal field theories, Phys. Lett. B212:451 (1988).

3. A. A. Belavin, A. M. Polyakov and A. B. Zamolodchikov, Infinite conformal symmetry in two-dimensional quantum field theory, Nucl. Phys. B241:333 (1984).

4. V. G. Knizhrik and A. B. Zamolodchikov, Current algebra and Wess-Zumino model in two dimensions, Nucl. Phys. B247:83 (1984)

5. See, for example, L. Faddeev, Integrable models in (1+1)-dimensional quantum field theory, in: "Recent Advances in Field Theory and Statistical Mechanics" (Le Houches 1982), J.-B. Zuber and R. Stora, eds., Elsevier Science Pub., (1984).

6. A. A. Belavin and V. G. Drinfel'd, Solutions of the classical Yang-Baxter equation for simple Lie algebras, Funkts. Anal. i Ego Prilozhen. 16:1 (1982).

7. T. Kohno, Hecke algebra representations of braid groups and classical YangBaxter equations, Adv. Stud. in Pure Math. 16:255 (1988).

8. T. Kohno, Quantized universal enveloping algebras and monodromy of braid groups, Nagoya Univ. preprint (1989).

9. M. Kato and P. Valtancoli, unpublished.

10. See, for example, A. N. Kirilloy and N. Yu. Reshetikhin, Representations of the algebra $U_{q}(s l(2))$, $q$-orthogonal polynomials and invariants of links, LOMI preprint E-9-88 (1988).

11. A. Tsuchiya and Y. Kanie, Vertex operators in conformal field theory on $P^{1}$ and monodromy representations of braid group, Adv. Stud. in Pure Math. 16:297 (1988). 